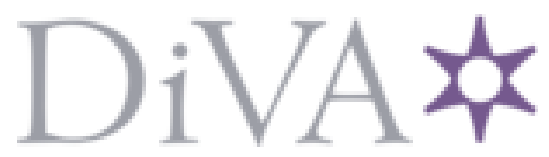

http://www.diva-portal.org

This is the published version of a paper presented at International Conference on Automation Science and Engineering (CASE), 2014 IEEE.

Citation for the original published paper:

Khodabakhshian, M., Feng, L., Wikander, J. (2014)

Predictive control of the engine cooling system for fuel efficiency improvement.

In:

N.B. When citing this work, cite the original published paper.

Permanent link to this version:

http://urn.kb.se/resolve?urn=urn:nbn:se:kth:diva-170377 


\title{
Predictive Control of the Engine Cooling System for Fuel Efficiency Improvement
}

\author{
M. Khodabakhshian, L. Feng and Jan Wikander
}

\begin{abstract}
The engine cooling system in trucks is one of the main sources of parasite load. Thus fuel efficiency can be improved by optimal control of engine thermal management system considering fuel consumption minimization as the objective. Although several optimal control methods have been proposed for the engine cooling system, their main emphasize is on regulating engine and coolant temperature in an acceptable range rather than minimizing fuel consumption. In contrast, this paper investigates the fuel saving potential of predictive optimal control methods for the engine cooling system of conventional trucks. Our method exploits the idea of energy buffers in the automotive system, where the engine cooling system and the battery serve as energy buffers. The advantages of this approach are the recovery of brake energy and the balance of energy sources so that the total energy loss is minimized. A model predictive controller is used as the real time controller, and the results are compared with a simple state feedback controller and a global optimal solution obtained by dynamic programming. The results show limited but notable improvement in fuel efficiency. The results also construct a base for ongoing research on energy buffer control in conventional heavy trucks.
\end{abstract}

\section{INTRODUCTION}

The main concerns in transportation industry are reducing fuel consumption and emissions. It is important to address the issues in both passenger cars and heavy trucks. Fuel is contributing to more than $30 \%$ of operational cost in trucks [1]. Different methods are being used to improve fuel efficiency in trucks. Besides the ongoing research on engine optimization, different new technologies have been introduced in the last decade. For example, hybridization has been proved to be a promising way for fuel efficiency improvement; yet, the hybridization process is usually very costly, thus the price for hybrid vehicles compared to conventional vehicles is high.

Another possibility to improve fuel efficiency is to control and minimize the energy being consumed by auxiliary subsystems such as braking, cooling, power steering, etc. Potential capabilities of auxiliary subsystems in decreasing fuel consumption have already been shown in [2] and [3]. The current trend of reducing the energy loss in auxiliaries is electrification. For example, the conventional mechanical coolant pump and radiator fans are gradually replaced by electric machines with variable speed [4], [5].

Another important method for reducing fuel consumption is to manage energy flow in the vehicle. Energy management is a hot research area in the hybrid electric vehicles, but it is

M. Khodabakhshian, L. Feng and J. Wikander are with KTH Royal Institute of Technology, Brinelvägen 85, Stockholm 10044, Sweden (+46704351617;e-mail: \{mokk,lfeng,janwi\}@kth.se). not discussed extensively in the conventional trucks. In hybrid electric vehicles (HEV) two (or more) energy buffers provide energy for different subsystems of the vehicle as well as propulsion of the vehicle. Similar to HEVs, different energy buffers can be recognized in conventional trucks. These energy buffers are mainly battery, vehicle mass (kinetic energy) and engine and coolant (thermal energy). Basically, the only energy source in the vehicle is fuel. However, energy buffers can store some amount of energy. For example, the vehicle has kinetic energy when moving, so it can be considered as an energy buffer.

Engine and the coolant circulating in the engine cooling system can be considered as an energy buffer if a larger operational range is allowed. Allowing higher temperature of coolant and engine within the safety window reduces the energy consumption of the cooling system. This is an advantageous strategy for fuel reduction when the engine is at low-efficiency operating point. The high temperature will be decreased when the engine is at high-efficiency operating point or even in the braking mode.

The idea of treating different systems in the vehicle as energy buffers and then managing them in a central controller is not discussed in detail, to the knowledge of authors. Ideas of holistic energy buffer control in trucks can be a way to increase fuel efficiency to considerable extents without extra cost for changing the architecture of drivetrain. Selection of proper optimal strategy to control the energy flow in the truck is the core of optimal energy buffer control. Ideas from similar fields can be used in this context. Energy management in HEV's as well as buildings can be implemented for energy buffer control in conventional trucks. A comprehensive survey of different energy management methods in HEVs is presented in [6]. It is important to consider the properties of each specific area, i.e. the energy management problem in buildings is similar to vehicle, but it is much more stable without many transient behaviors. In most of the literatures regarding engine cooling system management, the emphasis is on regulating engine temperature rather than fuel saving. The engine temperature regulation is of great importance. About $35 \%$ of the total chemical energy that enters an engine in the fuel is converted to useful crankshaft work, and about $30 \%$ of the fuel energy is carried away in the exhaust flow in the form of enthalpy and chemical energy [7]. Thus, about one third of the total energy must be dissipated to the surroundings by heat transfer. Removing the heat is highly critical in keeping an engine and engine lubricant from thermal failure. On the other hand, it is desirable to operate the engine as hot as possible to maximize thermal efficiency. Different strategies have been proposed for thermal management system such as 
classic state feedback controller [5],[8], optimal control methods [9], non-linear controllers [10],[11],[4], etc.

In the current work, the concept of energy buffer control in the conventional trucks is presented. The engine cooling system is then considered and a non-linear Model predictive controller (MPC) is presented to regulate the engine temperature considering fuel consumption minimization. Performance of the MPC controller is then compared to a global optimal control of the engine cooling system as well as a state feedback controller. Although improvement in fuel efficiency is rather limited, it shows the potential of fuel efficiency improvement of optimal energy buffer control.

This paper starts with a description of modelling of the truck and engine cooling system as well as a description of driving cycles in chapter II. Control strategy is presented in chapter III. In chapter IV, results from simulations and a discussion regarding the improvement potentials of presented controller is presented. The paper is concluded in chapter $\mathrm{V}$ by a conclusion.

\section{MODELING}

\section{A. Vehicle specification}

A conventional truck (4X2 tractor) FH 13 with electrified auxiliaries is used as the platform for analysis and simulations. Some of the vehicle specifications are presented in TABLE I.

TABLE I. SPECIFICATION OF TRUCK

\begin{tabular}{|l|l|}
\hline Component & Description \\
\hline Final drive ratio & 2.5 \\
\hline Wheel radius & $520 \mathrm{~mm}$ \\
\hline Frontal area: & $9.7 \mathrm{~m}^{\wedge} 2$ \\
\hline Drag coefficient & 0.53 \\
\hline Vehicle mass & $40000 \mathrm{~kg}$ \\
\hline Diesel engine & $12800 \mathrm{CC}$ \\
\hline Maximum Power output & $460 \mathrm{hp}, 1400-1900 \mathrm{rpm}$ \\
\hline Transmission & 12 speed Automatic gearbox \\
\hline Maximum Torque output & $2300 \mathrm{Nm}, 1400-1900 \mathrm{rpm}$ \\
\hline
\end{tabular}

\section{B. Longitudinal dynamics}

Longitudinal dynamics of the vehicle is described here. Main intention is to calculate the required power at each instant. Note that the battery is not included in the model and it is assumed that power demand for auxiliaries is directly added to the overall power demand from engine. Gear number is considered as an input to the system.

$$
P_{\text {dem }}=P_{\text {rol }}+P_{\text {aer }}+P_{\text {slp }}+P_{a c c}+P_{\text {aux }}
$$

where $P_{\text {dem }}$ is power demand from the engine, $P_{\text {rol }}$ is power required to overcome rolling resistance, $\mathrm{P}_{\mathrm{aer}}$ is power required to overcome aerodynamic force, $\mathrm{P}_{\text {slp }}$ is power required for climbing slopes, $\mathrm{P}_{\mathrm{acc}}$ is power equired for accelerating the vehicle and $\mathrm{P}_{\mathrm{aux}}$ is power required for auxiliaries. Each of demanded powers are calculated as,

$$
\mathrm{P}_{\mathrm{rol}}=v \cdot f \cdot m \cdot g \cdot \cos \alpha
$$

where $f$ is rolling resistant coefficient, $\alpha$ is gradient angle and $v$ is vehicle speed.

$$
P_{\text {aer }}=0.5 \cdot \rho \cdot c_{w} \cdot A \cdot v \cdot\left(v+v_{0}\right)^{2}
$$

where $c_{w}$ is drag coefficient, $A$ is frontal area and $v_{0}$ is headwind speed.

$$
\begin{gathered}
P_{\text {slope }}=m \cdot g \cdot v \cdot \sin \alpha \\
P_{\text {acc }}=m \cdot v \cdot \frac{d v}{d t} \\
P_{\text {aux }}=P_{p}+P_{f}
\end{gathered}
$$

$P_{p}$ and $P_{f}$ are demanded power for operating pump and fan respectively. Note that moment of inertia of engine, gearbox, shaft and wheels are neglected.

\section{Engine cooling system modeling}

The engine cooling system used in this study consists of an electric coolant pump, five identical electric fans and a 3-way valve. The valve is assumed to just have two states of open and close; however, during the simulations it is assumed that the thermostat is always open. The schematic of the engine cooling system is shown in Figure 1.

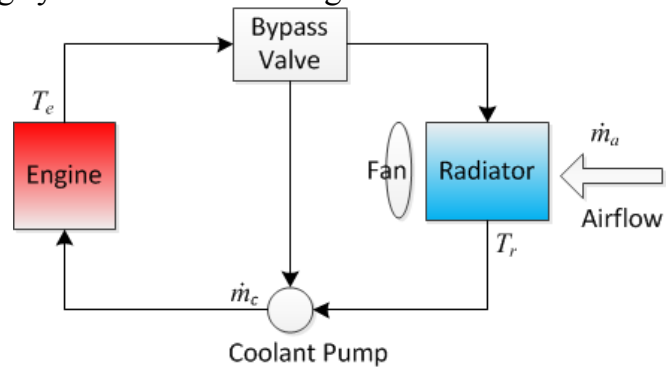

Figure 1. The Schematic of the Simplified Cooling System

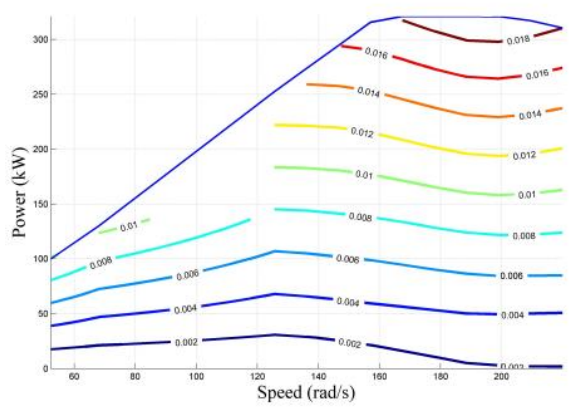

Figure 2. Engine map

The cooling system is modeled as a simple 2 states model. The state-space representation of the model is explained here. The control inputs are coolant mass flow rate through the pump and air mass flow rate through the radiator. The time delays in the system caused by, e.g., pipe length, are ignored. The heat transfer inside the engine is also ignored. It is assumed that the heat is only taken away from engine by coolant flow, so the effects of the air going around the engine, radiation and the heat taken away by exhaust are all ignored. The model has been used and verified in different papers [11], [4], [12]. Heat exchange equations are written using the second law of thermodynamics. The model can be described as:

$$
\begin{gathered}
\dot{T}_{e}=\frac{1}{C_{e}} Q_{i n}-\frac{c_{c}}{C_{e}} \dot{m}_{p}\left(T_{e}-T_{r}\right) \\
\dot{T}_{r}=\frac{\dot{m}_{p}}{C_{r}}\left(T_{e}-T_{r}\right)-\frac{\varepsilon c_{a}}{C_{r}} \dot{m}_{f}\left(T_{e}-T_{\infty}\right) \\
x=\left[\begin{array}{l}
T_{e} \\
T_{r}
\end{array}\right] \quad u=\left[\begin{array}{l}
\dot{m}_{p} \\
\dot{m}_{f}
\end{array}\right] \quad v=\left[Q_{i n}\right] \quad y=\left[\begin{array}{c}
T_{e} \\
T_{r}
\end{array}\right]
\end{gathered}
$$

where $T_{e}$ is engine temperature, $C_{e}$ is engine heat capacity, $c_{c}$ is coolant specific heat, $T_{r}$ is radiator temperature, $C_{r}$ is radiator heat capacity, $c_{a}$ is specific heat of air, $\varepsilon$ is constant, 
$\dot{m}_{p}$ is coolant flow in pump, $\dot{m}_{f}$ is air flow in radiator and $Q_{i n}$ is generated heat in engine. $Q_{i n}$ is calculated using the fuel rate map (Figure 2).

\section{Electric water pump}

The variable speed coolant pump is a centrifugal water pump driven by a DC motor. The equations describing the electric coolant pump are described here.

$$
\frac{d i_{p}}{d t}=\frac{1}{L_{p}}\left(V_{p}-R_{p} i_{p}-K_{E M F p} \omega_{p}\right)
$$

where $K_{E M F p}$ is back EMF in pump $(\mathrm{rad} / \mathrm{s}), V_{p}$ is pump voltage, $i_{p}$ is pump current, $R_{p}$ is pump electrical resistance, $\omega_{p}$ is pump speed and

$$
\frac{d \omega_{p}}{d t}=\frac{1}{J_{p}}\left(-\left(b_{p}+R_{f} V_{0}^{2}\right) \cdot \omega_{p}+K_{m p} i_{p}\right)
$$

where $R_{f}(P, Q)$ is nonlinear fluid resistance and $V_{0}$ is fluid volume per radian. $b_{p}$ is pump system viscous damping, $K_{m p}$ is pump torque constant, $J_{p}$ is moment of inertia in pump. We also have

$$
\dot{m}_{c}=\rho_{c} \cdot Q=\rho_{c} \cdot\left(2 \pi r_{1} b_{1} V_{r}\right)
$$

where $V_{r}$ is the inlet radial velocity component for the design point flow rate and is calculated as:

$$
V_{r}=r_{1} \omega_{p} \tan \beta_{1}
$$

where $b_{1}$ is inlet impeller width and $\beta_{1}$ is inlet impeller angle. By neglecting the dynamics in the pump and working out the equation, we will have

And

$$
P_{p}=\alpha \dot{m}_{c}
$$

$$
\alpha=\frac{\left(b_{p}+R_{f} V_{0}^{2}\right)}{\left(2 \pi \rho_{c} b_{1} r_{1}^{2} \tan \beta_{1}\right) K_{m p}}
$$

\section{E. Electric radiator fan}

Five identical electrical axial fans are used to cool the radiator. Each fan is driven by a DC motor with a PWM speed control. It is assumed that all of the fans are working with the same speed thus having similar power consumption and air flow rate. The fan air speed is a nonlinear function of the fan rotational speed. The formulation described here is based on [11]. Similar to the pump, equations for electrical fan are:

$$
\begin{gathered}
\frac{d i_{f}}{d t}=\frac{1}{L_{f}}\left(V_{f}-R_{f} i_{f}-K_{E M F f} \omega_{f}\right) \\
\frac{d \omega_{p}}{d t}=\frac{1}{J_{f}}\left(-B_{r} \omega_{f}+K_{f} i_{f}-\rho_{a} A_{f} R_{f} V_{a f}^{2}\right)
\end{gathered}
$$

$V_{a f}$ is fan air speed. It can be calculated as:

$$
V_{a f}=\left(\left(\frac{K_{f}}{\eta_{f} \rho_{a} A_{f}}\right) \cdot i_{f} \cdot \omega_{f}\right)^{0.3}
$$

$\eta_{f}$ is fan efficiency. The radiator air mass flow can also be calculated as:

$$
\dot{m}_{f}=\beta_{r} \rho_{a} A_{f} V_{a f}+\dot{m}_{r a m}
$$

$J_{f}$ and $B_{r}$ are equivalent moment of inertia and viscous damping of motor shaft and fan assembly. The power consumption for the fan can be calculated using the pressure difference in the two side of fan. The pressure increase can be calculated as

$$
\Delta P_{\text {fan }}=P_{\text {fan }, \text { out }}-P_{\text {fan }, \text { in }}
$$

$$
P_{f a n, 1}=\frac{\dot{m}_{f} \Delta p_{f a n}}{\rho_{a} \cdot \eta_{f a n}\left(\Delta p_{f a n}, \dot{m}_{f}\right)}
$$

where $\eta_{\text {fan }}$ is the combined efficiency of fan and DC motor. the efficiency can be calculated using a look up table. The overall power consumption of fans will be

$$
P_{\text {fan }}=N_{f} \cdot P_{\text {fan }, 1}
$$

where $N_{f}$ is number of fans.

\section{Driving cycle}

Since the engine cooling system is the focus of this study, it is important to choose proper driving cycle to see the effects of proposed strategy. Two different driving cycles are chosen in this study for simulation. One is a simple virtual cycle which consists of one uphill-downhill section and one acceleration-deceleration section (Figure 3). This cycle is used to understand the behavior of the system and to show the reason that the proposed controller increases fuel efficiency. Another driving cycle is a real route of $90 \mathrm{~km}$ between two cities in Sweden (Figure 4). This cycle is used to get quantitative results and verify the system performance. In the rest of the paper, the former cycle is named as simple cycle and the latter is called complex cycle.
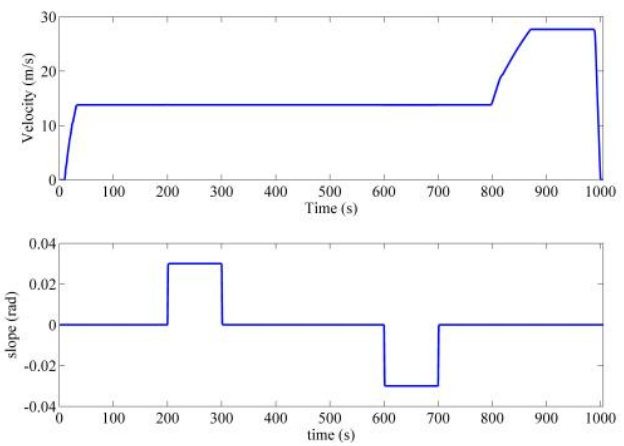

Figure 3. Simple virtual driving cycle
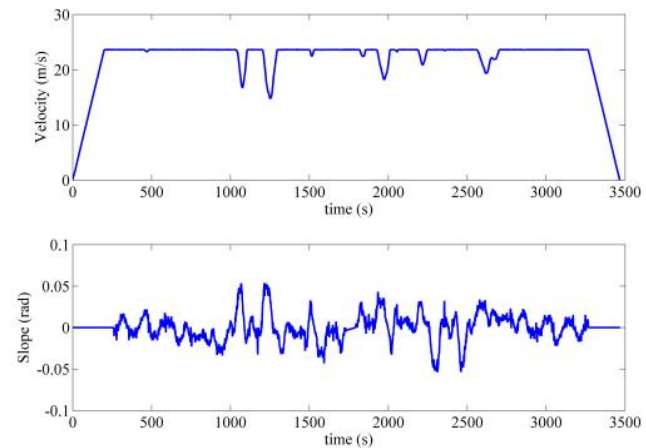

Figure 4.Complex real driving cycle

\section{CONTROLLER}

The control objective is to minimize the total fuel consumption for a given driving cycle. The general objective function can be written as

$$
\min _{u(t)} \int_{0}^{T} \dot{m}_{f}\left(\tau_{e}(u(t), d(t)), \omega_{e}(t)\right) d t
$$

subject to

$$
\begin{aligned}
& \dot{m}_{p, \text { min }} \leq \dot{m}_{p}(t) \leq \dot{m}_{p, \text { max }} \\
& \dot{m}_{f, \text { min }} \leq \dot{m}_{f}(t) \leq \dot{m}_{f, \text { max }}
\end{aligned}
$$


for $0<t<T$.

$$
\begin{gathered}
T_{e, \min } \leq T_{e}(t) \leq T_{e, \max } \\
T_{r, \min } \leq T_{r}(t) \leq T_{r, \max }
\end{gathered}
$$

where $u(t)$ is the same as $u$ in (9), $T$ is traveling time, $\tau_{e}$ is engine torque, $d(t)$ is the driving cycle signals which include speed and altitude and $\omega_{e}(t)$ is engine speed. In this paper, it is assumed that the vehicle exactly follows the driving cycle. The model of the system is explained in (7)-(9).

\section{A. Dynamic programming}

Dynamic programming (DP) is a well-known method for solving optimal control problems [13]. The solution found by DP is guaranteed to be globally optimal. DP is a numerical method which uses the decision making based on principle of optimality to sequence of decisions which together define an optimal policy and trajectory. In this paper, DP is used to construct a bench mark for evaluating real time controllers. The DPM tool [14] is used for simulation and the formulation of cost-to-go, etc. are explained in [14]. The state space model explained in (7)-(9) is discretized with time step of 1 second as

$$
\begin{aligned}
& T_{e}(k+1)=\frac{1}{C_{e}} Q_{i n}(k)-\frac{c_{c}}{C_{e}} \dot{m}_{p}(k) \cdot\left(T_{e}(k)-T_{r}(k)\right)+ \\
& T_{e}(k) \\
& \quad T_{r}(k+1)=\frac{\dot{m}_{p(k)}}{C_{r}}\left(T_{e}(k)-T_{r}(k)\right)-\frac{\varepsilon c_{a}}{C_{r}} \dot{m}_{f}(k) \cdot \\
& \left(T_{e}(k)-T_{\infty}\right)+T_{r}(k)
\end{aligned}
$$

Since the main intention of using DP is global optimization, computational load is not very important at this stage, so the grid for states and inputs is considered relatively small to guarantee optimality and decrease the error from interpolation.

\section{B. MPC controller}

A non-linear model predictive controller is used in this paper for real time control of engine cooling system. MPC is a powerful tool to handle optimal control problems with many constraints which makes it a proper tool to be used in the current work. The formulation of the MPC which is used in this paper is a repeated solution of a finite horizon optimal control problem with respect to system dynamics and constraints on states and control inputs. Similar method is used in [15] and [16]. MPC is explained in details in [17] and [18]. At each sampling instant, the following algorithm is performed.

- Calculating future trajectory

- Minimizing the cost function over the prediction horizon

- Applying the first member of control sequence

It is assumed that at time $t$, the future trajectory of the vehicle and consequently the future heat produced by engine is known. So the problem to be solved is

Subject to

$$
\min _{u(t)} \int_{t_{0}}^{t_{0}+t_{p}} \dot{m}_{f}\left(\tau_{e}(u(t), d(t)), \omega_{e}(t)\right) d t
$$

$$
\begin{aligned}
\dot{x}= & f(x, u, v) \\
& x \in X \\
& u \in U
\end{aligned}
$$

where $t_{0}$ is current time, $t_{p}$ is prediction time, $f$ is state space model and $X$ is feasible state, and $U$ is admissible controls. The problem is then rewritten as

subject to (24)-(27).

$$
V(k)=\sum_{i=H_{w}}^{H_{p}} \dot{m}_{f u e l}
$$

where $V$ is cost, $k$ is time step, $H_{w}$ is the start of the prediction horizon, which is considered 0 in this case, $H_{p}$ is prediction horizon, and the limits for constraints are selfexplanatory. The sampling time and discretization step is 1 second. The prediction horizon has direct effect on the performance of the controller and the computational load. To achieve desirable results, a prediction horizon of at least 20 seconds is necessary based on trial and error. The results presented in this paper are based on prediction horizon of 30 seconds. The problem is discretized similar to (28)-(29). However, the discretization of grid of the states and inputs has changed in order to reduce the computational load as much as possible. The fuel consumption can directly be calculated using the engine map. The cost function is minimized for the prediction horizon, and the first member of control sequence is applied to the system. The cost function is minimized using DP. Since DP is only applied for a limited horizon, the computational load at each step is not as heavy as a global optimization. Usage of DP is possible due to access to full prediction of upcoming driving situation which comes from a prediction unit.

\section{State feedback controller}

For better evaluation of the MPC controller, a simple state feedback controller is designed using pole-placement method. The controller tries to stabilize the engine and radiator temperatures to reference values. For designing the controller, the engine cooling system is linearized around the reference temperature, which is assumed constant in this case. This linearized system is

$\left\{\dot{x}=A x+B_{u} u+B_{v} v+F\right.$

$\left\{y=C x+D_{u} u+D_{v} v+G\right.$

where

$$
\begin{aligned}
& A=\left(\frac{\partial f}{\partial x}\right)_{\left(x_{0}, u_{0}, v_{0}\right)} B_{u}=\left(\frac{\partial f}{\partial u}\right)_{\left(x_{0}, u_{0}, v_{0}\right)} B_{v}=\left(\frac{\partial f}{\partial v}\right)_{\left(x_{0}, u_{0}, v_{0}\right)} \\
& C=\left(\frac{\partial g}{\partial x}\right)_{\left(x_{0}, u_{0}, v_{0}\right)} D_{u}=\left(\frac{\partial g}{\partial u}\right)_{\left(x_{0}, u_{0}, v_{0}\right)} D_{v}=\left(\frac{\partial g}{\partial v}\right)_{\left(x_{0}, u_{0}, v_{0}\right)} \\
& F=f\left(x_{0}, u_{0}, v_{0}\right)-A x_{0}-B_{u} u_{0}-B_{v} v_{0} \\
& G=g\left(x_{0}, u_{0}, v_{0}\right)-C x_{0}-D_{u} u_{0}-D_{v} v_{0} \\
& A=\left.\frac{\partial f}{\partial x}\right|_{\left(x_{0}, u_{0} v_{0}\right)}=\left[\begin{array}{ll}
\frac{\partial f_{1}}{\partial x_{1}} & \frac{\partial f_{1}}{\partial x_{2}} \\
\frac{\partial f_{2}}{\partial x_{1}} & \frac{\partial f_{2}}{\partial x_{2}}
\end{array}\right]_{\left(x_{0}, u_{0} v_{0}\right)}= \\
& {\left[\begin{array}{cc}
-\frac{c_{c}}{C_{e}} \dot{m}_{p} & \frac{c_{c}}{c_{e}} \dot{m}_{p} \\
\frac{c_{c}}{c_{r}} \dot{m}_{p}-\frac{\varepsilon c_{a}}{c_{r}} \dot{m}_{f} & -\frac{c_{c}}{C_{r}} \dot{m}_{p}
\end{array}\right]_{\left(x_{0}, u_{0} v_{0}\right)}} \\
& B_{u}=\left.\frac{\partial f}{\partial u}\right|_{\left(x_{0}, u_{0} v_{0}\right)}=\left[\begin{array}{ll}
\frac{\partial f_{1}}{\partial u_{1}} & \frac{\partial f_{1}}{\partial u_{2}} \\
\frac{\partial f_{2}}{\partial u_{1}} & \frac{\partial f_{2}}{\partial u_{2}}
\end{array}\right]_{\left(x_{0}, u_{0} v_{0}\right)}= \\
& {\left[\begin{array}{cc}
\frac{1}{C_{e}} \cdot \frac{\partial F_{2}}{\partial \dot{m}_{p}}-\frac{c_{c}}{C_{e}}\left(T_{e}-T_{r}\right) & \frac{1}{C_{e}} \cdot \frac{\partial F_{2}}{\partial \dot{m}_{f}} \\
\frac{c_{c}}{C_{r}}\left(T_{e}-T_{r}\right) & -\frac{\varepsilon c_{a}}{C_{r}}\left(T_{e}-T_{\infty}\right)
\end{array}\right]_{\left(x_{0}, u_{0} v_{0}\right)}}
\end{aligned}
$$


$B_{v}=\left.\frac{\partial f}{\partial v}\right|_{\left(x_{0}, u_{0} v_{0}\right)}=\left[\begin{array}{ll}\frac{\partial f_{1}}{\partial v_{1}} & \frac{\partial f_{1}}{\partial v_{2}} \\ \frac{\partial f_{2}}{\partial v_{1}} & \frac{\partial f_{2}}{\partial v_{2}}\end{array}\right]_{\left(x_{0}, u_{0} v_{0}\right)}=$

$\left[\begin{array}{cc}\frac{\partial F_{2}}{\partial \omega_{e n g}} & \frac{\partial F_{2}}{\partial T_{e}} \\ 0 & 0\end{array}\right]_{\left(x_{0}, u_{0} v_{0}\right)}$

$C=\left.\frac{\partial g}{\partial x}\right|_{\left(x_{0}, u_{0} v_{0}\right)}=\left[\begin{array}{ll}\frac{\partial g_{1}}{\partial x_{1}} & \frac{\partial g_{1}}{\partial x_{2}} \\ \frac{\partial g_{2}}{\partial x_{1}} & \frac{\partial g_{2}}{\partial x_{2}}\end{array}\right]_{\left(x_{0}, u_{0} v_{0}\right)}=\left[\begin{array}{ll}1 & 0 \\ 0 & 1\end{array}\right]_{\left(x_{0}, u_{0} v_{0}\right)}$

$D_{u}=\left.\frac{\partial g}{\partial u}\right|_{\left(x_{0}, u_{0} v_{0}\right)}=\left[\begin{array}{ll}\frac{\partial g_{1}}{\partial u_{1}} & \frac{\partial g_{1}}{\partial u_{2}} \\ \frac{\partial g_{2}}{\partial u_{1}} & \frac{\partial g_{2}}{\partial u_{2}}\end{array}\right]_{\left(x_{0}, u_{0} v_{0}\right)}=\left[\begin{array}{ll}0 & 0 \\ 0 & 0\end{array}\right]_{\left(x_{0}, u_{0} v_{0}\right)}$

$\left(x_{0}, u_{0} v_{0}\right)$ are equilibrium points. We also have

$F_{2}=Q_{\text {in }}=f\left(\omega_{\text {eng }}, T_{d r v}, \dot{m}_{f}, \dot{m}_{r}, V_{\text {veh }}\right)$

Then the poles of the system are identified and the controller tries to put the poles in the required positions. The system has two poles and one of them is very close to the unstable region and should be moved in order to guarantee stability of the system. The proper value for the new location of the poles is decided based on trial an error and studying the behavior of system. The gains for the controller are calculated using Matlab control toolbox. This controller is called simple controller in the rest of the paper.

\section{SIMULATION AND RESULTS}

The required inputs for the described thermal model in section III were provided by simulation of the complete longitudinal dynamic vehicle model in Autonomie [19] which is a Matlab/Simulink based software. Autonomie is developed by Argonne National Laboratory in collaboration with General Motors (GM).

\section{A. Global optimization}

Initial results from the simulation using dynamic programming and the simple controller are presented here. In the control result of dynamic programming, a drop in the engine temperature can be noticed before the two situations of high power demand from the engine; at 200 seconds and 800 seconds as can be seen in Figure 5. This helps the engine to avoid operating in low efficiency regions by pre-cooling the engine in the situations in which power demand from the engine is not high, and thus causing less power demand for pump and fans in the high power demand situations. This contributes to fuel saving in the vehicle. Note that the boundaries on the engine temperature and the temperature gradient of coolant are not corresponding to the real case, but the behavior is similar.

During the simulations, an improvement in the fuel consumption can be noticed when comparing the simple controller and the global optimal controller. As can be seen in the Figure 5, the simple controller temperature has a small offset compare to the global optimal controller. It is because the maximum temperature of the engine has been assumed as a hard constraint, so the maximum temperature should not rise above that. To see the effect of pre-cooling, another simulation has been performed, in which both controllers are following the maximum possible engine temperature, but the simple controller can go higher in some cases $(200-400,600-$ $800)$. Even in this case, an improvement of $0.37 \%$ can be seen. This implies the potential of fuel consumption improvement using optimized control.

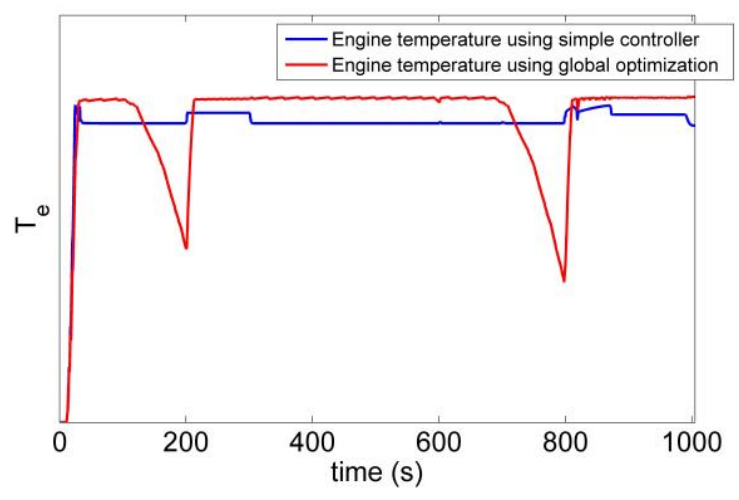

Figure 5. Engine temperature using global optimization and simple controller

\section{B. Real time controller}

Results from implementing MPC controller is presented here. Since the cost function is minimized using DP, the behavior which is expected from global optimization problem can also be seen here. An important factor which should be taken into account is the fact that if the prediction horizon is chosen too long, the computational burden in some points will be increased to an extent which makes the controller unreasonable to be used as a real time controller. On the other hand, if the prediction horizon is chosen too short, the controller cannot manage the energy flow in the buffer, and the pre-cooling effect cannot be seen. Using different predication horizon in the simulations, it was seen that a prediction horizon of at least 20 seconds is required to achieve favorable results. The results presented here are obtained using 30 seconds as prediction horizon. The grids for states and inputs had to be reduced in order to reduce computational burden. Engine temperature from implementing real time controller is given in Figure 6 for the first 300 seconds, which shows similar behavior to the global optimal controller. Fuel consumption of the vehicle using global optimization, real time controller using MPC and real time controller using state feedback controller in simple and complex driving cycles is presented in TABLE II.

\section{CONCLUSION}

A nonlinear model predictive control for optimal control of the engine cooling system considering fuel consumption reduction and temperature regulation has been presented. The idea of energy buffer control in conventional vehicles is described to show the potential fuel efficiency improvement of optimal energy flow management using prediction.

Results have been compared to a global optimal controller as well as a simple state feedback controller to show the performance of the model predictive controller using prediction. The presented controller shows satisfactory result for fuel efficiency reduction and engine temperature regulation. The results show better performance compared to 
the state feedback controller and comparable result with the global optimal controller.

In this paper, only the engine cooling system has been considered. Consideration of other energy buffers e.g. battery is the subject of ongoing research. The practical issues regarding controller implementation in a real vehicle are also not considered. Finally, the computational complexity of the controller has not been fully investigated, and there is a room for major improvement in the algorithms used in the predictive controller, especially the dynamic programming algorithm.

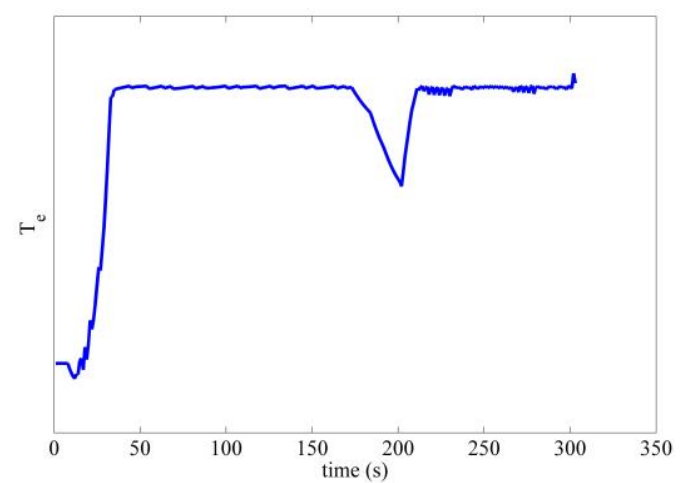

Figure 6. Engine temperature trajectory in the first 300 seconds

TABLE II. FUEL CONSUMPTION IN DIFFERENT CYCLES USING DIFFERENT CONTROLLERS

\begin{tabular}{|l|l|l|l|}
\hline controller & DP & $\begin{array}{l}\text { MPC } \\
\text { (increase in } \\
\text { fuel } \\
\text { consumption) }\end{array}$ & $\begin{array}{l}\text { Simple } \\
\text { (increase in } \\
\text { fuel } \\
\text { consumption) }\end{array}$ \\
\hline simple & 28.91 & $28.97(0.2 \%)$ & $29.12(0.7 \%)$ \\
\hline complex & 41.92 & $42.1(0.4 \%)$ & $42.35(\sim 1 \%)$ \\
\hline
\end{tabular}

\section{ACKNOWLEDGMENT}

This work is financially supported by the EU FP7 project COmplete Vehicle Energy-saving Technologies for HeavyTrucks (CONVENIENT).

\section{REFERENCES}

[1] O. Arici, J. H. Johnson, and A. J. Kulkarni, "The Vehicle Engine Cooling System Simulation Part 1 Model Development," SAE technical paper 1999-010240 , Mar. 1999.

[2] M. Khodabakhshian, J. Wikander, and L. Feng, "Fuel efficiency improvement in HEVs using electromechanical brake system," in 2013 IEEE Intelligent Vehicles Symposium (IV), 2013, pp. 322327.

[3] C. Andersson, "On auxiliary systems in commercial vehicles," PhD Dissertation, Department of industrial electrical engineering and automation Lund University, 2004.

[4] P. Setlur, J. R. Wagner, D. M. Dawson, and E. Marotta, "An Advanced Engine Thermal Management System: Nonlinear Control and Test,"
IEEE/ASME Trans. Mechatronics, vol. 10, no. 2, pp. 210-220, Apr. 2005.

[5] J. R. Wagner, V. Srinivasan, D. M. Dawson, and E. E. Marotta, "Smart Thermostat and Coolant Pump Control for Engine Thermal Management Systems,", SAE technical paper 2003-01-0272, Mar. 2003.

[6] A. Sciarretta and L. Guzzella, "Control of hybrid electric vehicles," IEEE Control Syst. Mag., vol. 27, no. 2, pp. 60-70, Apr. 2007.

[7] J. B. Heywood, Internal combustion engine fundamentals. New York: McGraw-Hill, 1988.

[8] E. Cortona and C. H. Onder, "Engine Thermal Management with Electric Cooling Pump," SAE technical paper 200-01-0965, Mar. 2000.

[9] A. Al Tamimi, M. Salah, and A. Al-Jarrah, "Neural Network-based Optimal Control for Advanced Vehicular Thermal Management Systems," SAE technical paper, 2011-01-2184, Sep. 2011.

[10] P. Setlur, J. Wagner, D. Dawson, and J. Chen, "Nonlinear controller for automotive thermal management systems," in Proceedings of the 2003 American Control Conference, 2003., 2003, vol. 6, pp. 4699-4704.

[11] M. H. Salah, T. H. Mitchell, J. R. Wagner, and D. M. Dawson, "Nonlinear-Control Strategy for Advanced Vehicle Thermal-Management Systems," IEEE Trans. Veh. Technol., vol. 57, no. 1, pp. 127-137, Jan. 2008.

[12] E. C. KANNE, "Engine Thermomanagement for Fuel Consumption Reduction,", PhD Dissertation, Swiss federal institute oftechnology, 2000.

[13] D. P. Bertsekas, Dynamic Programming and Optimal Control. Athena Scientific, 2012.

[14] O. Sundstrom and L. Guzzella, "A generic dynamic programming Matlab function," in IEEE International Conference on Control Applications, 2009, no. 7, pp. 1625-1630.

[15] D. Maxime, "Predictive Energy Management for Hybrid Electric Vehicles - Prediction Horizon and Battery Capacity Sensitivity," IFAC Symposium Advances in Automotive Control 2010, pp. 270-275.

[16] H. A. Borhan, A. Vahidi, A. M. Phillips, M. L. Kuang, and I. V. Kolmanovsky, "Predictive energy management of a power-split hybrid electric vehicle," in 2009 American Control Conference, 2009, pp. 3970-3976.

[17] J. M. Maciejowski, Predictive control with constraint. Essex: Pearson Education Limited, 2002.

[18] A. Bemporad, "Model Predictive Control Design: New Trends and Tools," in Proceedings of the 45th IEEE Conference on Decision and Control, 2006, no. 1, pp. 6678-6683.

[19] Autonomie, "http://www.autonomie.net/," 2012. 